\title{
Appearance of Negative Differential Conductivity in Graphene Nanoribbons at High-Harmonics
}

\author{
Musah Rabiu ${ }^{1}$, Samuel Y. Mensah ${ }^{2}$, Sulemana S. Abukari ${ }^{2}$ \\ ${ }^{1}$ Department of Applied Physics, University for Development Studies, Navrongo, Ghana \\ ${ }^{2}$ Physics Department, Center for Laser and Fiber Optics, University of Cape Coast, Cape Coast, Ghana \\ Email: mrabiu@uds.edu.gh
}

Received February 8, 2013; revised March 11, 2013; accepted April 3, 2013

Copyright (C) 2013 Musah Rabiu et al. This is an open access article distributed under the Creative Commons Attribution License, which permits unrestricted use, distribution, and reproduction in any medium, provided the original work is properly cited.

\begin{abstract}
We theoretically study current dynamics of graphene nanoribbons subject to DC-AC driven fields. We show that graphene exhibits negative differential conductivity (NDC) at high-harmonics. NDC occurs in the neighborhood where a constant electric field is equal to amplitude of ac field. We also observe NDC at both even and odd harmonics and at wave mixing of two commensurate frequencies. The even harmonics are more pronounced than the odd harmonics. A possible use of the present method for generating terahertz frequencies at even harmonics in graphene is suggested.
\end{abstract}

Keywords: Graphene Nanoribbon; Energy Spectrum; Electronic Conductivity; Terahertz; Negative Differential Conductivity

\section{Introduction}

Graphene has attracted much attention since its discovery in 2004 by Geim and his team [1]. The great interest in studying graphene is driven by its unique band structure [2] and many unusual physical properties like electrical, thermal and mechanical properties [3]. Despite these, there have been difficulties in realizing graphene-based electronic devices, probably, the fact that the graphene sheet lacks band gap, edge defects, disorder, etc. However, various attempts have been made to introduce $2 \mathrm{D}$ graphene sheet, for the purpose of overcoming some of these challenges, into a $1 \mathrm{D}+$ quantization. This phenomenon results into graphene nanoribbon (GNR) which is assumed as an unrolled single-wall carbon nanotube (SWCNT).

Depending on the nature of the nanoribbon edges, one gets two symmetry groups; armchair graphene nanoribbon (aGNR) and zigzag graphene nanoribbon (zGNR). Armchair and zigzag GNRs show metallic or semicon-ducting electronic properties [4] depending on the number of dimer rows, $\mathcal{N}$ along transverse direction. $\mathcal{N}$ is related to the width of the nanoribbon as $W=\sqrt{3} a(\mathcal{N}-1)=2$ [5]. Electron dynamics of both ribbons have different properties, mostly due to the berry phase and pseudo spin [6]. Edge states have significant contribution to graphene properties, because in a nano-meter size ribbon, massless
Dirac fermions can reach the edges within a femto-second before encountering any lattice effects, and avoiding electron-electron interaction, eletron-phonon interaction, etc.

In this paper, we employ Boltzmann transport equation based on relaxation time approximation to study negative differential conductivity (NDC) in GNRs. In conventional semiconductor devices including semiconductor superlattices, an NDC behavior is known to offer great potential for high frequency applications as Bloch oscillators, frequency multipliers, and fast switching devices. For this reason, the NDC effect has been greatly explored and discussed in several graphene nanostructures, particularly in [7]. NDC can also be observed in other graphene allotropes; carbon nanotubes (CNT) [8]. The unique energy spectrum of holes and electrons in GNRs, especially its narrow gap nature leads to nontrivial features such as NDC in the THz regime [7].

The rest of this paper is organized as follows; In Section 2, we derived the electronic current density of aGNR and zGNR and imposed certain conditions to reduce the equations to forms appropriate for our model. The results obtained in Section 3 are discussed in great details in Section 4. The paper finally concludes in Section 5 where some recommendations for future applications are made. 


\section{The Theory}

Following the approach of refs, [8,9] for SWCNT and semiconductor superlattices, we consider an undoped GNR (both zigzag and armchair) exposed simultaneously to $\mathrm{DC}+\mathrm{AC}$ electric field

$$
E(t)=\sum_{j=0}^{n} E_{j} \mathrm{e}^{\mathrm{i}\left(\omega_{j} t+\alpha_{j}\right)}
$$

which is seen as a superposition of $n$ harmonic waves polarized along one direction with angular frequency. The phase difference between the $(j+1)$ th and $j$ th component being $\alpha_{j+1}-\alpha_{j}=\alpha$ is arbitrary, $j$ is an integer. $E_{j}$ are the field amplitudes. We require that

$\omega_{0}=\alpha_{0}=0$. This will correspond to the DC component of the applied field. The dynamics of free $\pi$-electrons in graphene can be describe by the time-dependent Boltzmann transport equation (BTE) based on relaxation time approximation in zero magnetic field as

$$
\frac{\partial f(k, t)}{\partial t}+\frac{e E(t)}{\hbar} \frac{\partial f(k, t)}{\partial k}=\Gamma\left[f(k, t)-f_{0}(k)\right] .
$$

We also assume a spatial uniform graphene nanoribbon. Again, the inverse of the relaxation time $\Gamma$ is momentum independent. In Equation (1), $f_{0}(k)$ and $f(t, k)$ are the equilibrium and non-equilibrium Fermi electron distribution functions, respectively. $e$ is the electronic charge, $k$ is the electron wave vector and $\hbar$ is the reduced plank constant. In the following section, we will calculate electronic current density for GNRs.

\subsection{Armchair and Zigzag Nanoribbon Band Structures}

The energy band structure of aGNR and zGNR is characterized by three parameters; band index $\lambda$, phase $\theta$ and wave vector $k \quad[5,6]$. For aGNR,

$$
\mathcal{E}^{\lambda}(k, \theta)=\lambda \gamma_{0} \sqrt{1+4 \cos ^{2}(s \Delta \theta)+4 \cos (s \Delta \theta) \cos (k l)},
$$

and for zGNR,

$$
\mathcal{E}^{\lambda}(k, \theta)=\lambda \gamma_{0} \sqrt{1+4 \cos ^{2}\left(k l^{\prime}\right)+4 \cos (s \Delta \theta) \cos \left(k l^{\prime}\right)} \text {. }
$$

where $\lambda= \pm 1$ with $(+)$ for conduction band and ( -$)$ for valence band. $l=\sqrt{3} a / 2$ and $l^{\prime}=a / 2, a$ is the lattice spacing which has numerical value of $0.246 \mathrm{~nm}$, $\gamma_{0} \sim 3.0 \mathrm{eV}$ is the overlap integral and $\theta$ is the phase perpendicular to the quasi-momentum $\hbar k$. The $1 \mathrm{BZ}$ of aGNR is bounded by $k l=[-\pi / 2, \pi / 2]$ and the zGNR is $k l^{\prime}=[0, \pi] . \quad k$ is parallel to the edge and has translational symmetry along this direction. For aGNR, the transverse wave vector (phase) is quantized according to the rule [6] $\theta_{s}=s \Delta \theta$ with $\Delta \theta=\pi /(\mathcal{N}+1)$ and $s=1,2, \cdots, \mathcal{N}$. Unlike aGNR, the nature of transverse wave vector quantization is complicated in zGNR, de- pending on both $k$ and $\theta$ as $\Delta \theta_{s}=(\pi j+\Lambda(k, \theta)) /(\mathcal{N}+1)$. However, for simplicity we assume $\Lambda$ is constant, say $\pi / 2$, so that $\Delta \theta_{s}=(2 j+1) \pi /(2(\mathcal{N}+1))$. Except this little subtlety for zGNRs, all that will be discussed in the following for aGNR are equally applied to the zGNR.

Now, we employed the translational invariance of graphene ribbon in the reciprocal space and expand in Fourier series functions $f, f_{0}$ and $\mathcal{E}$ along the edge having periodicity in $k$.i.e.,

$$
\begin{aligned}
& f_{0}(k, \theta)=\sum_{r \neq 0} f_{r}(\theta) \mathrm{e}^{\mathrm{i} r k l}, \\
& f(k, \theta, t)=\sum_{r \neq 0} f_{r}(\theta) \mathrm{e}^{\mathrm{i} r k l} \Phi_{r}(t), \\
& \mathcal{E}(k, \theta)=\gamma_{0} \sum_{r \neq 0} \mathcal{E}_{r}(\theta) \mathrm{e}^{\mathrm{i} r k l} .
\end{aligned}
$$

The Fourier coefficient $f_{r}$ is expressed as

$$
\begin{aligned}
f_{r}(\theta)= & \sum_{s=1}^{\mathcal{N}} f_{r s} \Delta \theta \delta\left(\theta_{s}-s \Delta \theta\right) \text { with } \\
& f_{r s}=\frac{l}{\pi s \Delta \theta} \int_{-\pi / 2 l}^{\pi / 2 l} \mathrm{~d} k f_{0}(k, \theta) \mathrm{e}^{-i r k l}, f_{r s}=f_{-r s}^{*}
\end{aligned}
$$

and

$$
\mathcal{E}_{r}=\frac{l}{2 \pi \gamma_{0}} \int_{-\pi / 2 l}^{\pi / 2 l} \mathrm{~d} k f_{0}(k, \theta) \mathrm{e}^{-\mathrm{i} r k l}, \mathcal{E}_{r}=\mathcal{E}_{-r}^{*} .
$$

In (6), $s$ counts the number of dimers $\mathcal{N}$ in the GNRs. The factor $\Phi_{r}$ in the equation is a central point in this paper and so has to be determined. $r$ is an integer and not equal to zero. We consider a classical limit in which energy levels could be excited due to thermal fluctuations, i.e. $\Delta \mathcal{E} \square K_{B T} \square \mathcal{E}_{C}$. This condition is also necessary for large enough field, so that charge carriers can escape low energy scattering [10]. The energy level spacing $\Delta \mathcal{E}=\pi W \gamma_{0} l / A, \mathcal{E}_{C}$ is the charging energy, $k_{B}$ is Boltzmann constant, $T$ is lattice temperature and $W$ is the graphene nonoribbon width. In what follows, we will compute $\Phi(t)$. To do this, (5), (6) and (7) are substituted in (1) following the simplification scheme $j=j^{\prime}=1,2,3, \cdots \quad$ and $m_{j}, n_{j}= \pm 1, \pm 2, \pm 3, \cdots$. We obtained

$$
\Phi_{r}(t)=\sum_{n_{j}, v_{j}=-\infty}^{\infty} \prod_{j=1}^{n} J_{n_{j}}\left(\beta_{j}\right) J_{n_{j}-v_{j}}\left(\beta_{j}\right) \times \frac{\mathrm{e}^{\mathrm{i} v_{j} \omega_{j} t+\mathrm{i} v_{j} \alpha_{j}}}{1+\mathrm{i} \tau\left(\beta_{0}+v_{j} \omega_{j}\right)} .
$$

where $v_{j}=n_{j}-m_{j}$.

\subsection{Sheet Current Density}

The sheet current density of graphene can be determined from the relation

$$
j(t)=\frac{g_{s} g_{s}}{A} \sum_{k} e v_{k} f_{k} .
$$


the sheet area $A=W L . g_{s}, g_{v}$ are the spin and valley degeneracies, respectively. The current density can also be written as

$$
j(t)=\frac{g_{s} g_{v} e}{4 \pi^{2}} \sum_{s=1}^{\mathcal{N}} \int \mathrm{d} k v\left(k, \theta_{s}\right) f\left(k, \theta_{s}, \Phi_{\mathrm{r}}\right) .
$$

the velocity of Dirac fermions in graphene is defined as $v(k)=\partial \mathcal{E} / \hbar \partial k$. In terms of the Fourier coefficients,

$$
v(k, \theta)=\frac{\mathrm{i} \gamma_{0} l}{\hbar} \sum_{r \neq 0} \mathcal{E}_{r} \mathrm{e}^{\mathrm{i} r k l}
$$

Giving

$$
j(t)=\mathrm{i} \sum_{r=1}^{\infty} j_{0, r} \Phi_{r}(t)+c . c
$$

with

$$
j_{0, r}=\frac{2 g_{s} g_{v} e \gamma_{0}}{\pi l \hbar} \Delta \theta \sum_{s=1}^{\mathcal{N}} \mathcal{E}_{r s} f_{r s}, \quad j_{0, r}^{*}=-j_{0,-r} .
$$

Note the $r$ dependence of $\beta_{j}, \beta_{0}$ and the summation over the index in (10). Using the formalism by Litvinov and Manasson [9]. Equation (14) can be put in a Taylor-like expansion in terms of $E_{j}$. i.e.,

$$
j=j_{D C}+\frac{1}{2}\left[\sum_{j} E_{j} \sum_{v_{j} \neq 0} \sigma_{n_{j} \omega_{j}}+C . C\right] \mathrm{e}^{\mathrm{i} v_{j} \omega_{j} t}+\cdots .
$$

where

$$
j_{D C}=\sum_{r=1}^{\infty} j_{0, r} \sum_{-\infty}^{\infty} \prod_{j=1}^{n} J_{n_{j}}^{2}\left(\beta_{j}\right) \frac{\mathrm{i}+\beta_{0} \tau+n_{j} \omega_{j} \tau}{1+\left[\tau\left(\beta_{0}+n_{j} \omega_{j}\right)\right]^{2}}+C . C
$$

is the differential DC current density (for $v_{j}=0$ ), and

$$
\begin{aligned}
\sigma_{n_{j} \omega_{j}}= & 2 \sum_{r=1}^{\infty} j_{0, r} \sum_{n_{j}=-\infty}^{\infty} \prod_{j=1}^{n} \frac{J_{n_{j}}\left(\beta_{j}\right) J_{n_{j}-v_{j}}\left(\beta_{j}\right)}{E_{j}} \\
& \times \frac{\mathrm{i}+\beta_{0} \tau+n_{j} \omega_{j} \tau}{1+\left[\tau\left(\beta_{0}+n_{j} \omega_{j}\right)\right]^{2}} \mathrm{e}^{v_{j} \alpha_{j}}
\end{aligned}
$$

is the large-signal dynamic nonlinear conductivity at $v_{j}$ harmonic with drive frequency $\omega_{j}$.

\section{Negative Differential Conductivity}

\subsection{Pure DC Limit}

To see immediately that (16) demonstrates NDC, we consider a pure DC limit where $\omega_{j} \rightarrow 0$. The Bessel functions except the $n=0$ term will vanish. The real part of the differential conductivity $\sigma(0)=\lim _{\omega_{j} \rightarrow 0} \partial \mathrm{j} / \partial E_{0}$ become

$$
\sigma(0)=\sum_{r=1}^{\infty} \sigma_{0, r} \frac{e l \tau}{\hbar} \frac{1-\left(\beta_{0} \tau\right)^{2}}{\left[1+\left(\beta_{0} \tau\right)^{2}\right]^{2}}
$$

So that if $\beta_{0}>\tau^{-1}$, the differential conductivity is negative and NDC is manifest in GNRs. Electron dynamics may be come more complicated in the presence of high-frequency components in addition to the static electric fields. High negative differential conductivity thus may result in GNRs if an external drive force is applied.

\subsection{Monoharmonics}

If one component of the AC field in (2) is applied, then $n=1$ and (16) simplifies to

$$
j=\sum_{r} j_{0, r} \sum_{n=-\infty}^{\infty} J_{n}^{2}(\beta) \frac{\beta_{0} \tau+n \omega_{1} \tau}{1+\left[\tau\left(\beta_{0}+n \omega_{1}\right)\right]^{2}},
$$

after dropping the subscript on $n$. Here, it not clear immediately how NDC can be seen. To observe the phenomenon, we plot $j$ with $E_{0}$ for $\omega \tau \square 1$ as shown in Figure 1 (top) for armchair ribbon and in Figure 1 (buttom) for zigzag ribbon,
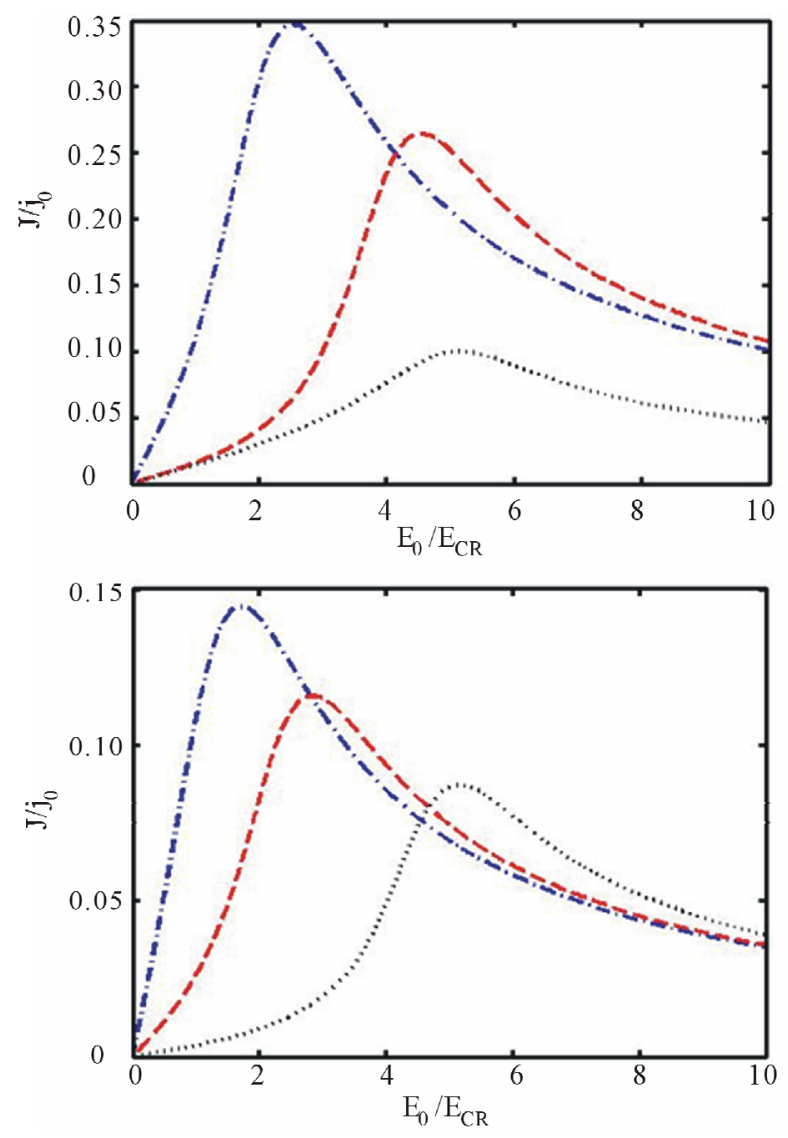

Figure 1. Negative differential conductivity for (top) armchair and (buttom) zigzag graphene nanoribbons at different AC field amplitudes. Blue-dashed-dot: $E=4 E_{c r}$, Reddashed: $E=4 E_{c r} \cdot \omega \tau=0.1$. The onset of NDC is at low static fields around $E_{0} \sim E$. It departs from this condition at high fields. 
The effect of NDC is strong in zigzag graphene than in armchair graphene. Especially, in the limit of high harmonic field, NDC is greatly suppressed in aGNR.

\subsection{Biharmonics}

In practical situations, one can also allow graphene nanoribbon subject to two $\mathrm{AC}$ field components. In that case, we let $j=1,2$ in our general formalism in Section 2 . Equation (15) takes the form

$$
\begin{aligned}
j= & \mathrm{i} \sum_{r=1}^{\infty} j_{0, r} \sum_{n_{1}, n_{2}=-\infty}^{\infty} \sum_{v_{1}, v_{2}=-\infty}^{\infty} J_{n_{1}}\left(\beta_{1}\right) J_{n_{1}-v_{1}}\left(\beta_{1}\right) \\
& \times J_{n_{2}}\left(\beta_{2}\right) J_{n_{2}-v_{2}}\left(\beta_{2}\right) \frac{\mathrm{e}^{\mathrm{i} v_{1} \alpha_{1}+\mathrm{i} v_{2} \alpha_{2}}}{1+\mathrm{i} \tau\left(\beta_{0}+n_{1} \omega_{1}+n_{2} \omega_{2}\right)},
\end{aligned}
$$

and from which (17) follows. We have eliminated the time dependence by averaging over the period of the fields to find the time-independent current $j$. In the left hand side, we replaced $\langle j(t)\rangle=j$, and in the right hand side a delta function emerges which ensures that $v_{1}=-\left(\omega_{2} / \omega_{1}\right) v_{2}$. If $\omega_{1}=\omega_{2}$, then one must put $\alpha_{1}=0$ and $\alpha_{2}=\alpha$ so that $v_{1}=v_{2}$. However, we shall generalize this to a case of commensurate frequencies. We exemplified the case by a biharmonic having frequencies which can be periodic $\omega_{2}=\mu \omega_{1}$ or non-periodic, $\omega_{2} \neq \mu \omega_{1}$ with $\mu=1,2, \cdots$. These two cases were studied in $[11,12]$ for semiconductor superlattices. Simplifying further, we linearize with respect to one of the field amplitudes (say, $E_{2}$ ). For a week field, $\beta_{2} \square 1$ and $J_{n}(\beta) \approx(\beta / 2)^{2} / n !$, which allows us to take $n_{2}\left(n_{2}-v_{2}\right)= \pm 1(0, \pm 1)$. Finally, the AC current density becomes

$$
\begin{aligned}
j= & \frac{e l \tau^{2}}{\mu \hbar} E_{2} \cos \alpha \sum_{r=1}^{\infty} j_{0, r} \frac{\beta_{0} \tau+n \omega \tau}{1+\left(\beta_{0} \tau+n \omega \tau\right)^{2}} \\
& \times J_{n}\left(r \beta_{1}\right)\left\{J_{n ? \mu}(r \beta)-J_{n+\mu}(r \beta)\right\} .
\end{aligned}
$$

$\beta_{1,2}=e r l E_{1,2} / \hbar \omega_{1,2}$. Equation (21) reduces to the monoharmonic case when $\mu=1$.

The nature of the NDC is observed for a simultaneously varying harmonic field and phase difference in a three dimensional plot shown in Figure 2.

\section{Discussion and Conclusions}

In Figure 1 normalized current density $j / j_{0}$ is plotted against reduced static electric field $E_{0} / E_{c r}$ for aGNR (left) and zGNR (right) for an applied AC + DC field. At low fields up to $E_{0}\left(j_{\max }\right)$, the quantum derivative of the $j-E_{0}$ characteristic yields a positive slope. A negative slope results for $E_{0}>E_{0}\left(j_{\max }\right)$. In the whole region, $E_{0}>E_{0}\left(j_{\max }\right)$ graphene nanoribbon device will operate under Negative Differential Conductivity (NDC). A consequence of NDC in GNR is a formation of electric field domains that impedes a continuous motion of electrons generated by the external electric field and thus blocks high frequency oscillations in the nanoribbon. NDC disappears quite faster in aGNR as $E \rightarrow \infty$ compared to zGNR which is more rubust at this limit.

The curves in Figure 3 demonstrate NDC phenomenon. They are obtained at wave mixing of two commensurate frequencies, $\omega_{2}=\mu \omega_{1}$. Figure 3 (left), $\mu=$ odd and Figure 3 (right), $\mu$ even . The onset of NDC in oddharmonics occurs around $E_{0} \sim E_{1}$, and in even-harmonics it starts at $E_{0} \leq E_{1}$. In both cases, as in the previous NDC graphs, $\omega \tau \square 1$.

The combined effect of phase shift $\alpha$, between two AC fields and amplitude on NDC is depicted in Figure 2. There are three peaks at low bias fields at points $\left(E \sim E_{c r}, \alpha=0\right),\left(E \sim E_{c r}, \alpha=\pi\right)$ and $\left(E \sim E_{c r}, \alpha=2 \pi\right)$. It is not very clear what these crests and troughs mean, they might be associated with field domains along one direction (for $\alpha=0,2 \pi, \cdots$ ) and others along the opposite direction (for $\alpha=\pi, 3 \pi, \cdots$ ) and vice-versa. This means that electron velocity is higher in the domain with the same polarization along the electron direction and lower in the domain with opposite polarization.

In conclusion, we have demonstrated that graphene nanoribbons exhibit NDC regions in its $j-E_{0}$ characteristics at low bias field when $\omega \tau \square 1$. NDC is observed either in the presence of bias field alone or by superimPosing AC fields on the bias field. For one AC field component, NDC occurs around $\omega \tau \sim 0.1$. When two $\mathrm{AC}$ fields at commensurate frequencies are applied, highharmonic NDC emerge for both even and odd harmonics at rather very low frequencies $\omega \tau \sim 0.01$. The evenseries gives pronounced high-harmonic NDC than the odd-series. The presence of high-harmonic NDC means that it is possible for high-frequency generation in graphene nanoribbons when electric field domains are suppressed at high enough applied frequencies $\omega \tau \square 1$ and $E_{0}>E_{c r}$. We therefore suggest this approach for the

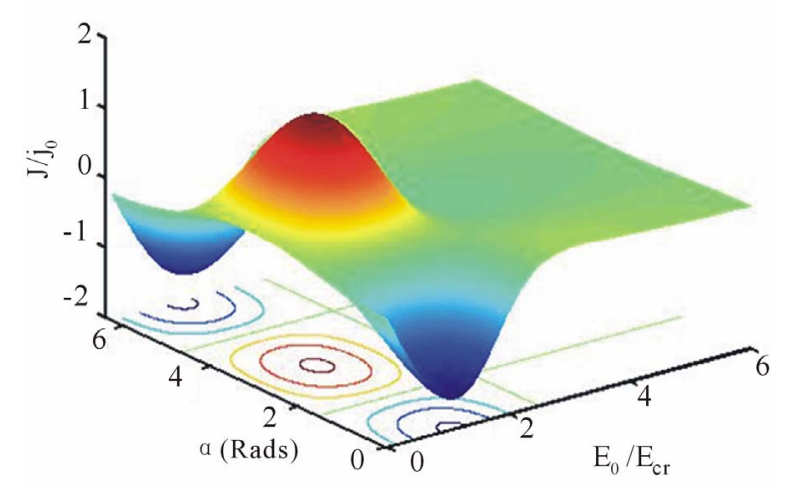

Figure 2. Negative differential conductivity of aGNR due to applied static field amplitude, $E_{0}$ and phase shift, $\alpha$ between two AC fields. 

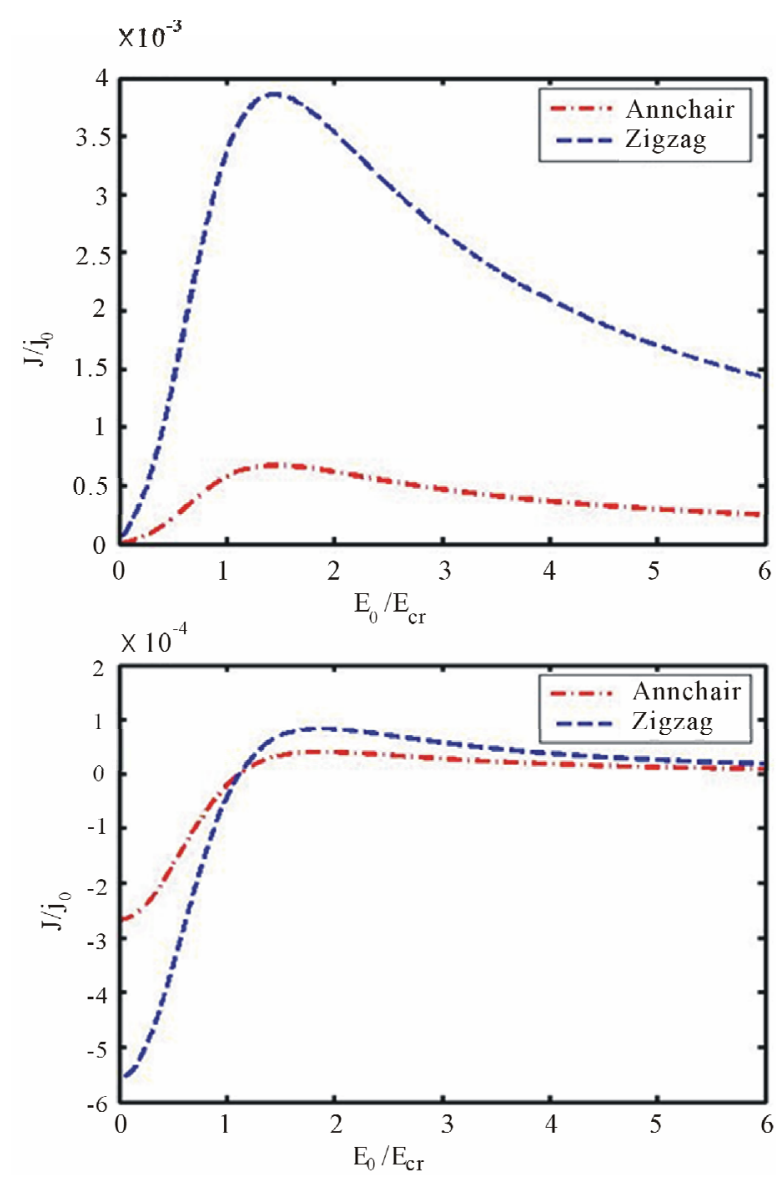

Figure 3. Negative differential conductivity due to wave mixing of two AC field amplitudes. (top) $\mu=e v e n$ and (buttom) $\mu=$ odd . The parameters used are $E_{1}=0.2 E_{c r}$, $E_{2}=E_{c r}$ and $\omega \tau=0.01$.

study of terahertz generation in graphene.

\section{REFERENCES}

[1] K. S. Novoselov, A. K. Geim, S. V. Morozov, D. Jiang, Y. Zhang, S. V. Dubonos, I. V. Grigorieva and A. A. Firsov, "Electric Field Effect in Atomically Thin Carbon Films," Science, Vol. 306, No. 5696, 2004, pp. 666-669. doi:10.1126/science. 1102896

[2] K. S. Novoselov, A. K. Geim, S. V. Morozov, D. Jiang, M. I. Katsenelson, I. V. Grigorieva, S. V. Dubonus and A. A. Firsov, "Two-Dimensional Gas of Massless Dirac Fermions in Graphene," Nature, Vol. 438, No. 7065, 2005, pp. 197-200. doi:10.1038/nature04233
[3] R. S. Shishir and D. K. Ferry, "Intrinsic Mobility in Graphene," Journal of Physics: Condensed Matter, Vol. 21, No. 23, 2009, Article ID: 232204. doi:10.1088/0953-8984/21/23/232204

[4] L. Brey and H. A. Fertig, "Electronic States of Graphene Nanoribbons," Physical Review B, Vol. 73, No. 23, 2006 , Article ID: 235411.

[5] F. Hipolito, A. H. Chaves, R. M. Ribeiro, M. I. Vasilevskiy, V. M. Pereira and N. M. R. Peres, "Enhanced Optical Dichroism of Graphene Nanoribbons," Physical Review $B$, Vol. 86, No. 11, 2012, Article ID: 115430. doi:10.1103/PhysRevB.86.115430

[6] K. Sasaki, K. Kato, Y. Tokura, K. Oguri and T. Sogawa, "Theory of Optical Transitions in Graphene Nanoribbons," Physical Review B, Vol. 84, No. 8, 2011, Article ID: 085458. doi:10.1103/PhysRevB.84.085458

[7] V. Ryzhii, M. Ryzhii and T. Otsiji, "Negative Dynamic Conductivity of Graphene with Optical Pumping," Journal of Applied Physics, Vol. 101, No. 8, 2007, Article ID: 083114. doi:10.1063/1.2717566

[8] S. S. Abukari, S. Y. Mensah, K. W. Adu, N. G. Mensah, K. A. Dompreh, A. Twum, C. L. Y. Amuah, M. Amekpewu and M. Rabiu, "Domain Suppression in the Negative Differential Conductivity Region of Carbon Nanotubes by Applied AC Electric Field," World Journal of Condensed Matter Physics, Vol. 2, No. 4, 2012, pp. 274 277. doi:10.4236/wjcmp.2012.24045

[9] V. I. Litvinov and A. Manasson, “A Large-Signal Negative Dynamic Conductivity and High-Harmonic Oscillations in a Superlattice," Physical Review B, Vol. 70, No. 19, 2004, Article ID: 195323. doi:10.1103/PhysRevB.70.195323

[10] S. K. Sekwao and J. P. Leburton, "Hot Electron Terahertz Oscillations in Graphene: Crater and Terraces in the Carrier Distribution Function," Preprint, arXiv:1003.0842v1.

[11] K. A. Pronin and A. D. Bandrauk, "Coherent Control of Electric Currents in Superlattices and Molecular Wires: Effect of Relaxation," Physical Review B, Vol. 69, No. 19, 2004, Article ID: 195308. doi:10.1103/PhysRevB.69.195308

[12] K. Seeger, "High-Frequency-Induced Phase-Dependent DC Current by Bloch Oscillator Non-Ohmicity," Applied Physics Letters, Vol. 76, No. 1, 2000, pp. 82-84. doi:10.1063/1.125663 